\title{
Quaderni
}

QUADERNI Communication, technologies, pouvoir

68 | Hiver 2008-2009

Militantisme médical et fabrique des politiques de santé

\section{L'émergence des soins palliatifs dans la médecine, une forme particulière de militantisme de fin de vie}

The Emergence of Palliative Care in Medicine

Michel Castra

\section{OpenEdition}

\section{Journals}

Édition électronique

URL : http://journals.openedition.org/quaderni/260

DOI : 10.4000 /quaderni.260

ISSN : 2105-2956

Éditeur

Les éditions de la Maison des sciences de l'Homme

Édition imprimée

Date de publication : 5 janvier 2009

Pagination : 25-35

\section{Référence électronique}

Michel Castra, «L'émergence des soins palliatifs dans la médecine, une forme particulière de

militantisme de fin de vie », Quaderni [En ligne], 68 | Hiver 2008-2009, mis en ligne le 05 janvier 2012,

consulté le 30 avril 2019. URL : http://journals.openedition.org/quaderni/260 ; DOI : 10.4000/

quaderni.260 


\section{$D$ ossier}

\section{l'émergence des}

soins palliatifs

\section{dans la médecine,}

une forme particulière

de militantisme de fin de vie

Michel

Castra

Maître de conférences Institut de sociologie et d'anthropologie Université de Lille 1 Laboratoire du Clersé - CNRS
$\mathrm{Au}$ cours des dernières décennies, les conditions du rapport à la mort se sont profondément transformées dans le champ de la médecine.

Les innovations thérapeutiques, l'invention de techniques de maintien en survie (réanimation, respiration et alimentation artificielles) et plus largement l'émergence de la fin de vie comme période distincte et susceptible d'une intervention spécifique ont contribué à redéfinir les pratiques et les conceptions des professionnels. De même, les préoccupations nouvelles liées à l'autonomie, à l'information et aux droits des malades ont largement participé à l'ensemble de ces évolutions qui permettent de mieux comprendre le développement d'une médecine palliative particulièrement représentative de ces mutations.

Ce texte entend rendre compte d'une partie du travail de recomposition des normes et des valeurs à l'œuvre dans la médecine avec la constitution d'une discipline spécialisée dans la prise en charge des patients confrontés à la mort ${ }^{1}$. Nous retraçons dans ce qui suit le processus de reconnaissance médicale et sociale des soins palliatifs en montrant que l'émergence de cette catégorie de « fin de vie » n'a été possible que par un double mouvement de revendications mené auprès de la société civile et des pouvoirs publics pour le développement de cette approche médicalisée du mourir, mais aussi en direction des professionnels de santé à travers un travail constant de légitimation. Si cette activité de persuasion emprunte les formes classiques de construction d'une expertise médicale (à travers notamment la maîtrise des douleurs et des symptômes de fin de vie) ou de constructions éthiques, elle prend aussi la forme d'un travail d'argumentation mené 
quotidiennement par les équipes mobiles de soins palliatifs qui s'emploient à imposer et à légitimer des standards de pratiques propres à la discipline auprès des équipes curatives de l'hôpital.

\section{Les soins palliatifs : un mouvement social à l'intérieur de la médecine}

Si dans les années 1970, la mort et la question de la prise en charge des malades mourants apparaît de plus en plus comme un sujet de préoccupation et de débat public, il n'existe pas à proprement parler de prise en charge standardisée et routinisée de la fin de vie. Chaque service hospitalier, en fonction du type de mort et de trajectoires de fin de vie prédominant (Glaser ; Strauss, 1968), adopte une prise en charge médicalisée qui dépend largement des orientations, des compétences et des savoir-faire des médecins et en particulier du chef de service. L'inexistence d'une pratique médicale stabilisée et reconnue dans ce domaine illustre le profond désintérêt du monde médical pour cette catégorie de patients non reconnus et en marge du monde hospitalier. En témoigne, le très faible nombre de publications médicales consacrées aux soins terminaux durant cette période.

Dans la seconde moitié des années 1970, une poignée de médecins, proches des milieux catholiques, découvre l'existence des soins palliatifs en Grande-Bretagne où il existe depuis plusieurs décennies une tradition de soins et d'assistance aux mourants (avec le mouvement des hospices). Les visites de l'Hospice Saint-Christopher de Londres (créé par Cicely Saunders en 1967), lieu emblématique de la médecine palliative moderne, puis au Canada de la première unité de soins palliatifs intra-hospitalière au monde, ouverte à l'hôpital Royal Victoria de Montréal en 1975, sont le prélude à l'engagement dans cette discipline naissante. La découverte de ces réalisations novatrices conduit ces pionniers à un changement radical de perspective tant du point de vue des valeurs et des thérapeutiques que des modèles d'organisation du travail ; la fin de vie apparaît alors de manière assez confuse comme un champ d'innovation possible. Ces médecins, pour la plupart, sont amenés à reconsidérer de manière assez radicale leur propre pratique. Cette réorientation s'effectue cependant de façon très empirique et pragmatique : dans leurs services respectifs, chacun procède à des essais, à des tentatives pour appliquer les méthodes de soulagement de la douleur et en particulier le maniement des morphiniques. C'est autour de ces nouvelles thérapeutiques antalgiques, perçues comme une opportunité, que s'élabore un consensus et qu'émerge une communauté d'intérêt autour de la fin de vie et des patients terminaux. Les médecins disposent alors de ressources efficaces qui permettent de renouveler assez rapidement les discours et les pratiques, et finalement de proposer un modèle original de prise en charge du mourir.

Il faut souligner ici que les soins palliatifs constituent une innovation un peu particulière dans la médecine, dans la mesure où elle se situe à contre-courant des logiques de scientificité, de technicité et de recherche qui caractérisent l'ensemble des progrès médicaux contemporains. Par ailleurs, cette innovation apparaît d'autant moins légitime aux yeux des autres médecins qu'elle s'inscrit à rebours du paradigme curatif et qu'elle prend pour objet l'échec même de la 
médecine : l'absence de guérison associée à la survenue d'une mort prochaine et son cortège de souffrances.

Définissant la fin de vie comme un domaine à part entière, ces professionnels introduisent une coupure transversale dans l'ordre des spécialités en accordant une place et une attention nouvelles au malade en fin de vie dans le monde médical. En revendiquant la fin de vie comme un problème médical à part entière, les pionniers des soins palliatifs s'engagent dans un processus de médicalisation d'une nouvelle forme de gestion du mourir qui prend la voie de la constitution d'un segment au sein de la profession pour se développer (Baszanger, 1995 ; Strauss, 1992). Le mouvement d'émergence de cette médecine palliative s'observe dans un premier temps par la dénonciation de la gestion médicalisée du mourir. Cette contestation des conditions inacceptables de la mort en milieu hospitalier s'inscrit dans une stratégie de distanciation et de différenciation qui constitue un des traits caractéristiques des processus de segmentation (Strauss, 1984). À travers la désignation de morts déviantes (souffrance, abandon du mourant, pratiques d'euthanasie clandestines...), le segment opère une régulation à la fois professionnelle et sociale, en venant signifier les prescriptions normatives nécessaires à une bonne prise en charge. L'affirmation d'un domaine de compétences et le développement d'une rhétorique sur les besoins du mourant vont également être au cœur du processus de segmentation : le développement d'une «revendication d'utilité» vise à gagner une légitimité à l'intérieur du monde médical. Si la question de la relation au patient fait l'objet d'une attention significative des praticiens dès l'origine de la discipline ${ }^{2}$, l'expertise de la douleur et des symptômes de fin de vie peut être considérée comme la pierre angulaire de la stratégie de distinction scientifique : il s'agit désormais de constituer la réalité de la fin de vie comme un problème strictement médical susceptible de relever de compétences et de savoirs techniques (Castra, 2003). C'est en particulier le maniement des morphiniques et l'utilisation des antalgiques majeurs qui vont être au cœur de ce travail de légitimation. Les professionnels des soins palliatifs s'efforcent de lutter contre les représentations négatives et les préjugés attachés à la morphine et à son utilisation dans la profession médicale. Par ailleurs, dénoncer le déficit de formation des médecins et la sous-utilisation notoire de la morphine en France permet aux promoteurs de la discipline d'accentuer leur positionnement comme innovateur.

Ces éléments techniques et thérapeutiques ne restent cependant pas confinés à l'arène médicale mais prennent rapidement pied dans la controverse sociale qui se développe en France dès 1984 autour de l'euthanasie volontaire. L'émergence d'une revendication pour le droit à mourir (menée notamment pas l'Association pour le Droit de Mourir dans la Dignité) est en effet saisie comme une opportunité pour les entrepreneurs d'une médecine palliative de rendre visible l'existence d'une gestion médicalisée du mourir. Acteurs majeurs du débat social sur la fin de vie, ces médecins se positionnent comme porte-parole légitimes de la profession médicale sur la scène publique en proposant une voie alternative entre acharnement thérapeutique et euthanasie.

Le segment des soins palliatifs, comme le souligne A. Strauss à propos du changement profes- 
sionnel dans la médecine, peut être analysé par analogie avec les mouvements sociaux dans la mesure où il possède une idéologie propre, des missions spécifiques, des formes d'organisation et d'action collective visant à renforcer leur position (Strauss, 1992). Ce militantisme palliatif s'inscrit dans un double travail de mobilisation : d'une part à l'intérieur de la communauté médicale où il s'agit d'établir la fin de vie comme période autonome et de circonscrire une place légitime dans l'espace médical appelant une expertise spécifique et d'autre part à l'extérieur de la médecine, en direction de la société civile et des autorités publiques qu'il importe de sensibiliser et de convaincre.

La réussite de cette entreprise innovante et la constitution d'un nouveau domaine disciplinaire tiennent en particulier à la capacité à mobiliser des alliés et à organiser cette revendication collective. L'émergence des soins palliatifs doit notamment être rapportée à la création d'un mouvement associatif qui a largement contribué à élargir le champ des revendications : en orientant la question de la prise en charge de la fin de vie sur le terrain social (volonté de «changer les mentalités face à la mort» et de créer du lien social autour du mourant) il s'agit d'acquérir une légitimité renouvelée et de favoriser l'extension du problème dans l'opinion publique. Grâce à ces relais associatifs, les soins palliatifs sortent de la marginalisation de l'hôpital et ne sont plus seulement cantonnés au domaine professionnel mais apparaissent comme un phénomène plus vaste qui dépasse les préoccupations strictement médicales.

En quelques années, les promoteurs de cette médecine palliative parviennent ainsi à associer un cercle d'acteurs plus large : psychologues, infirmières, aides-soignantes, kinésithérapeutes, bénévoles s'impliquent dans la pratique des soins aux malades en fin de vie et concourent à l'élaboration de la doctrine du groupe et des conceptions du travail qui en découlent. Ces différents acteurs contribuent ainsi à faire exister cette médecine dans différents milieux professionnels et à engager des délibérations dans des arènes variées. Le domaine de l'éthique médicale apparaît de ce point de vue comme un des lieux privilégiés d'objectivation des revendications sociales.

\section{Promouvoir une éthique du «bien mourir»}

Avec l'apparition des soins palliatifs et leur intégration au monde hospitalier, on voit progressivements'édifier, durant les années 1990, une nouvelle conception de l'éthique médicale à l'approche de la mort. La démarche palliative se situe en effet dans un contexte de déplacement de l'objectif médical traditionnel : il ne s'agit plus de guérir un patient, si bien que la légitimité des nouveaux buts à atteindre n'apparaît plus évidente dans une optique strictement médicale. Les soins palliatifs constituent un mouvement dans la médecine, dont les promoteurs entendent participer à un changement des conceptions professionnelles face à la fin de vie et promouvoir de nouvelles formes de prise en charge des patients. Les principes d'action mobilisés s'inscrivent ainsi dans une volonté de réhabiliter les malades mourants dans le système de soins, d'apaiser les inconforts, les douleurs et les souffrances, et plus largement de prendre en compte l'expérience subjective des patients face à la maladie et à la mort. Les croyances et valeurs sur lesquelles repose 
l'émergence de ce nouveau modèle de gestion du mourir, doivent être recherchées dans l'idéal d'une mort acceptée et pacifiée qui prédomine le champ des soins palliatifs. Au travers de ces principes, on assiste également à une redéfinition de l'éthique médicale dans le domaine des phases avancées et terminales de la maladie.

Dès l'origine, les entrepreneurs de la discipline palliative se sont efforcés de placer le questionnement éthique au centre de leur démarche de soin. À cet égard, c'est bien dans la perspective d'un conflit de valeurs sur la scène sociale mais aussi à l'intérieur de la profession médicale que les soins palliatifs s'affirment. Conçus comme un modèle alternatif à l'acharnement thérapeutique et à l'euthanasie, il s'agit de définir les conceptions d'une fin de vie acceptable fondée sur des valeurs telles que le respect de la personne, la liberté du malade, son autonomie, sa qualité de vie, l'évitement de la souffrance... À travers ce réajustement, ce «glissement» éthique d'une médecine qui entend promouvoir de nouveaux objectifs, c'est une redéfinition des conceptions du « bien » pour le malade qui est ici à l'œuvre et qui marque un changement de légitimité de l'action médicale fondée non plus sur une rationalité scientifique et technique mais sur de nouvelles croyances dans la finalité de l'intervention médicale auprès des malades en fin de vie. Comme le souligne un des pionniers français des soins palliatifs, Maurice Abiven, «cette médecine doit fonctionner selon des règles éthiques qui lui sont propres; la principale de ces règles étant que la qualité de la vie qui reste à vivre à ce malade doit primer sur la durée de cette vie; règle qui est exactement à l'opposé de la règle éthique fondamentale de la médecine, où l'on doit toujours s'employer à ce que la durée de la vie soit prioritaire, même si cela devait se faire aux dépens, de la qualité de la vie» (Abiven, 1999).

L'ambition éthique sans cesse mise en avant par les promoteurs des soins palliatifs est à cet égard particulièrement significative d'un réaménagement des valeurs et des normes en situation de fin de vie. En témoignent les modifications apportées au code de déontologie médicale en 1995 puis en 2006 qui viennent consacrer les principes et la doctrine des soins palliatifs. L'article 37, qui ne concerne pas uniquementlespatientsmourants, stipule qu' « En toutes circonstances, le médecin doit s'efforcer de soulager les souffrances du malade par des moyens appropriés à son état et l'assister moralement. Il doit s'abstenir de toute obstination déraisonnable dans les investigations ou la thérapeutique et peut renoncer à entreprendre ou poursuivre des traitements qui apparaissent inutiles, disproportionnés ou qui n'ont d'autre objet ou effet que le maintien artificiel de la vie». En revanche, l'article 38 vise spécifiquement les malades en fin de vie et affirme les contenus de la doctrine palliative : "Le médecin doit accompagner le mourant jusqu'à ses derniers moments, assurer par les soins et mesures appropriés la qualité d'une vie qui prend fin, sauvegarder la dignité du malade et réconforter son entourage. Il n'a pas le droit de provoquer délibérément la mort $»^{3}$.

De même, la loi d'avril 2005, qui fait suite à l'«affaire Vincent Humbert», s'inscrit pleinement dans cette logique palliative du 
«laisser vivre sans faire mourir» et écarte ainsi toute dépénalisation de l'euthanasie (voie dans laquelle se sont engagés les Pays-Bas et la Belgique). Parmi les principes mis en avant par la loi, on peut évoquer différentes dispositions comme le refus de toute obstination thérapeutique déraisonnable ou la reconnaissance du traitement anti-douleur à double effet pour une personne en fin de vie (on accepte notamment le fait qu'un médecin prescrive une thérapeutique contre la douleur risquant d'avoir pour effet secondaire de faire mourir le malade mourant) ; mais surtout la loi propose un cadre pour définir les conditions de l'arrêt des soins demandé par le patient et introduit l'obligation de tenir compte des éventuelles directives anticipées formulées par celui-ci ${ }^{4}$.

Cette refonte de l'éthique médicale peut être analysée non seulement comme une renégociation des domaines de compétences internes à la profession mais aussi comme une opportunité pour que celle-ci puisse reconstituer les conditions d'une légitimité renouvelée dans le domaine de la prise en charge des malades en phase terminale (Paradeise, 1985). Elle traduit également l'aspiration à une plus grande autonomie pour les malades qui demeure cependant limitée et largement encadrée par la profession médicale. Au travers de la loi d'avril 2005 (qui s'inscrit d'ailleurs dans la continuité de la loi de juin 1999 sur les soins palliatifs), l'État vient ainsi confirmer cette logique médicale nouvelle comme mode privilégié de régulation de la fin de vie ; régulation déléguée et assumée par des spécialistes dotés d'un savoir spécifique et chargés d'administrer ou de contrôler la proximité de la mort.

\section{L'institutionnalisation de la médecine pallia- tive comme nouvelle forme d'action collective dans la médecine}

Si le domaine de l'éthique médicale peut être considéré comme un premier aboutissement du travail de conviction et de persuasion mené par les promoteurs de la discipline, le processus d'objectivation trouve d'autres traductions concrètes avec, par exemple, la mise en place de guides de bonnes pratiques ou encore la production de textes législatifs qui apparaissent comme l'expression d'un consensus autour de la gestion palliative de la fin de vie. L'institutionnalisation des soins palliatifs dans le système de santé constitue cependant une étape particulièrement importante pour le développement de la discipline. Non seulement parce que la création en France à partir de 1987 des premières structures spécialisées dans la prise en charge des mourants peut être considérée comme la reconnaissance d'une expertise médicale dans ce domaine, mais aussi parce quelle rend possible la mise en place de nouvelles pratiques routinisées et l'élaboration puis la stabilisation de nouveaux savoir-faire qui n'avaient pas pu être initiés dans les services d'origine. En quelques années cette dynamique d'institutionnalisation engendre une multiplicité de structures et de configurations institutionnelles : USP (Unités de soins palliatifs), EMSP (équipes mobiles de soins palliatifs), réseaux de soins palliatifs, lits identifiés (en services curatifs)... Les soins palliatifs recouvrent ainsi une grande diversité de situations et d'ancrages institutionnels, contribuant à faire de la discipline, une entité visible mais aux contours flous et peu stables, caractéristique sans doute inhérente à un monde social en transformation permanente et en 
évolution rapide.

Il faut souligner le rôle majeur d'une société savante, la SFAP (société française d'accompagnement et de soins palliatifs), créée en 1990, qui s'impose rapidement comme le porte-parole de la discipline et l'interlocuteur principal des pouvoirs publics en matière de soins palliatifs. Dirigée par des médecins et rassemblant l'ensemble des acteurs professionnels et bénévoles impliqués dans ce domaine, la SFAP mène des actions visant à susciter une prise de conscience et à sensibiliser aussi bien l'opinion publique, les autorités publiques que les professionnels de santé : contacts avec la presse, organisation d'études et de recherches sur la fin de vie, organisation de congrès, et surtout contacts réguliers avec le ministère de la santé. Entretenant des liens étroits avec les acteurs politiques et administratifs de la santé, elle participe pleinement au travail politique qui s'efforce de traduire une évidence collective partagée en énoncés législatifs, en dispositifs d'action, ou en guide de bonnes pratiques, conduisant à une résolution provisoire du conflit (Tournay, 2007). Les mesures politiques et législatives en faveur de soins palliatifs témoignent bien d'un consensus autour des soins palliatifs comme solution au problème médical de la fin de vie ; ainsi le texte final de la loi du 9 juin 1999 «visant à garantir le droit à l'accès aux soins palliatifs » est-il voté à l'unanimité au Sénat et à l'Assemblée Nationale $^{5}$. De même, l'intégration rapide des soins palliatifs au système de santé, l'introduction de la discipline dans le cursus des études médicales, les modifications successives du code de déontologie ou encore les recommandations médicales de l'ANAES sont autant de signes qui témoignent de la relative réussite du processus d'objectivation des revendications sociales et professionnelles. Relative réussite car à défaut de développer des connaissances biomédicales rigoureuses, une part significative de la médecine palliative se trouve en marge des critères habituels de la médecine scientifique et des standards qui y sont associés, comme le souligne un des rapports de l'ANAES précisant que « la littérature spécifique aux soins palliatifs est rarement fondée sur des études de bonne qualité méthodologique $\|^{6}$. C'est du même coup l'expérience clinique des médecins qui fonde la normalisation de ces pratiques et leur standardisation. On assiste ainsi à un mode de régulation scientifique qui passe par une période inaugurale de variation des connaissances et des pratiques non stabilisées au moment de l'émergence de la discipline. Cette situation aboutit progressivement à un accord collectif informel (visible notamment au travers des publications professionnelles, des enseignements universitaires) sur les pratiques médicales, les protocoles à suivre en ce qui concerne l'utilisation des morphiniques et antalgiques majeurs ou encore les principes liés à l'accompagnement et aux dimensions relationnelles du travail auprès des mourants et de leurs proches. Intervenant dans un second temps, les recommandations successives de l'ANAES peuvent être envisagées comme une entente formelle entre praticiens qui émerge d'un consensus sur les « bonnes pratiques » en vigueur dans la discipline ; ces recommandations constituent de ce point de vue un effort significatif de standardisation des compétences et des pratiques médicales (Vinck; Weisz, 2007).

Il reste que la reconnaissance et l'intégration de la pratique palliative dans les outils de gestion et les nomenclatures administratives apparaît comme 
un processus qui reste encore inabouti comme en témoigne par exemple la valorisation très partielle de cette activité médicale dans le PMSI.

De même, si la démarche des soins palliatifs peut constituer une pratique routinisée dans nombre de services hospitaliers, on ne peut pas véritablement parler d'uniformisation des logiques médicales face à la fin de vie en dépit de la promulgation des standards évoquée plus haut. À cet égard, il est intéressant d'observer le travail des équipes mobiles de soins palliatifs (EMSP) qui interviennent auprès des équipes des services curatifs de l'hôpital qui en font la demande, non pas pour mener une activité de soins en se substituant aux soignants du service, mais pour assurer un rôle de conseil et de formation auprès de ces derniers. Cette mission de consultance s'apparente à une véritable entreprise morale (Becker, 1985) dans la mesure où leur activité vise bien à promouvoir de nouvelles normes de soins et à redéfinir les modalités du travail médical à l'approche de la mort (Castra, 2007). Ces équipes peuvent être interprétées comme relevant d'un dispositif militant visant à faire évoluer les pratiques et à diffuser les nouveaux standards de la médecine de fin de vie. Reposant sur une logique du compromis, ces structures s'efforcent de mettre en œuvre une pratique palliative à géométrie variable en fonction des contraintes et des rationalités propres à chaque service. Elles constituent ainsi une forme organisée d'expertise collective aboutissant à l'établissement de consensus locaux et toujours renégociés.

L'émergence des soins palliatifs en France est significative d'une approche rénovée du mourir qui repose sur l'agencement de pratiques anciennes de soins et d'accompagnement des mourants, le recours à des techniques plus modernes de lutte contre les douleurs et symptômes de fin de vie, associées à une prise en charge de la détresse morale et psychologique des patients en fin de vie. Le développement de cette médecine est particulièrement représentatif d'une dynamique de changement professionnel qui ne peut être dissociée des transformations sociales du rapport à la fin de vie et à la mort. Dans cette perspective, l'analyse des mobilisations collectives dans ce domaine témoigne du passage des revendications sociales et professionnelles dans le domaine de l'action publique. Elle invite à considérer la part de militantisme moral inhérente à la construction d'une entité médicale nouvelle. Les textes de lois, les codes de déontologie, et le mouvement de standardisation de cette médecine constituent de ce point de vue un bon indicateur du rapport de force politique entre les différents acteurs militants évoluant dans le champ de la fin de vie. Ils illustrent un accord collectif, sans doute transitoire, sur les modalités de prise en charge de l'approche de la mort. 
$R \cdot E ́ \cdot F \cdot E \cdot R \cdot E \cdot N \cdot C \cdot E \cdot S$

M. ABIVEN, « Les soins palliatifs terminaux », Actualité et Dossier en Santé Publique, $\mathrm{n}^{\circ} 28$, 1999, pp. 18-19.

N. ALTER, L'innovation ordinaire, Paris, PUF, Coll. Sociologies, 2000.

I. BASZANGER, Douleur et médecine, la fin d'un oubli, Paris : Seuil, Coll. La couleur des idées, 1995.

H. BECKER, Outsiders. Études de sociologie de la déviance, Paris, Métailié, Coll. Observations, 1985.

M. CASTRA, Bien mourir. Sociologie des soins palliatifs, Paris, PUF, 2003.

M. CASTRA, «Les équipes mobiles de soins palliatifs, entre expertise et «entreprise morale» ", Sociologie Santé, $\mathrm{n}^{\circ} 27$, décembre 2007, pp. 115-130.

B. GLASER ; A. Strauss, Time for dying, Chicago, Aldine Publishing Company, 1968.

C. PARADEISE, « Rhétorique professionnelle et expertise », Sociologie du travail, vol. XXVII, $\mathrm{n}^{\circ} 1,1985$, pp. 17-31.

A. STRAUSS, «Social worlds and their legitimation processes», in N. Denzin, (Ed.), Studies in symbolic interaction, vol. 4, Greenwich/London: JAI Press INC, 1982, pp. 171-190.

A. STRAUSS, «Social worlds and their segmentation processes », in N. Denzin, (Ed.), Studies in symbolic interaction, vol. 5, Greenwich/London, JAI Press INC, 1984, pp. 123-139.

A. STRAUSS, La trame de la négociation - Sociologie qualitative et interactionnisme, Paris, L'Harmattan, 1992.
V. TOURNAY, « Comment inscrire durablement les revendications militantes, les pratiques sociales et les récits historiques? », in V. Tournay (ss. dir.), La gouvernance des innovations médicales, Paris, PUF, 2007.

D. VINCK, G. WEISZ, «Les mutations de l'action publique sanitaire. Quelle histoire et quelle place donner aux régimes de production des connaissances ? », in V. Tournay (ss. dir.), La gouvernance des innovations médicales, Paris, PUF, 2007. 
$\mathrm{N} \cdot \mathrm{O} \cdot \mathrm{T} \cdot \mathrm{E} \cdot \mathrm{S}$

1. La contribution présentée ici est issue d'une recherche menée sur les transformations sociales du rapport à la fin de vie et à la mort dans la médecine à travers l'émergence des soins palliatifs. Il s'agissait d'appréhender dans ce travail le processus de médicalisation de la fin de vie et d'envisager cette nouvelle préoccupation dans la médecine du point de vue des pratiques concrètes de soins à l'hôpital. Pour réaliser cette recherche, trois niveaux d'observation ont été menés : une analyse de contenu de la presse (spécialisée et quotidienne) sur la période 19701995, 112 entretiens semi-directifs auprès de professionnels et acteurs des soins palliatifs, et une période d'observation participante de neuf mois dans différentes structures de soins palliatifs.

2. On peut par exemple évoquer la théorie de la «douleur totale» de C. Saunders qui appréhende la souffrance du mourant dans toutes ses composantes biologiques, psychologiques, sociales et spirituelles. La subjectivité est au cœur même du modèle du bien mourir propre aux soins palliatifs. L'expérience de la personne en fin de vie représente en effet une dimension essentielle du travail relationnel et de la prise en charge du malheur par les professionnels. La souffrance et la rupture biographique que vit le malade constituent un moment propice au dévoilement de cette subjectivité qui devient alors disponible pour être travaillée (Castra, 2003).

3. Code de déontologie, articles R.4127-37 et R.412738 du code de la santé publique, 14 décembre 2006. L'évolution est ici notable, comparée à la version du code de déontologie de 1979 (article 20) : «Le médecin doit s'efforcer d'apaiser les souffrances du malade. Il n'y a pas le droit d'en provoquer délibérément la mort » (Décret du 28 juin 1979).
4. Cette dernière disposition sur les directives anticipées doit être soulignée car elle n'est pas sans rappeler le «testament de vie » dont l'ADMD (Association pour le Droit de Mourir dans la Dignité. L'influence de cette association militant pour la dépénalisation de l'euthanasie volontaire est ici manifeste. Les directives anticipées de cette loi ne reprennent cependant que certains principes. Si elles témoignent bien de la reconnaissance de la volonté du patient en fin de vie, cette reconnaissance demeure limitée puisque ces directives ne s'imposent pas au médecin.

5. France, Loi n 99-477 du 9 juin 1999 visant à garantir le droit à l'accès aux soins palliatifs, Journal officiel de la République Française, 10 juin 1999, pp. 8487-8489.

6. Agence Nationale d'Accréditation et d'Évaluation en Santé, Modalités de prise en charge de l'adulte nécessitant des soins palliatifs - Recommandations pour la pratique clinique, décembre 2002, p. 10. 
$R \cdot E ́ \cdot S \cdot U \cdot M \cdot E ́$

Cet article retrace l'émergence récente d'une discipline médicale : les soins palliatifs. Ils concrétisent une nouvelle manière d'appréhender la réalité sociale et professionnelle du mourir. Cette médecine spécialisée dans la prise en charge des malades en fin de vie est étroitement liée à l'autonomisation de cette période de la maladie présentée comme relevant d'une expertise spécifique. C'est ce mouvement de constitution d'une entité médicale particulière que l'article annalyse, en essayant de comprendre le processus de segmentation qui a permis l'ouverture d'un domaine de compétences au sein du monde médical. En traçant les contours d'un nouveau domaine d'intervention et d'une nouvelle pratique, les promoteurs des soins palliatifs cherchent à affirmer une définition commune de leur objet et l'instauration de normes et de valeurs de référence dans la prise en charge des situations terminales.

\section{The emergence of palliative care in medicine}

Our intention is to understand how a new medical entity, palliative care, focused upon the end of life and its problems was brought about. The question here is trying to work out a genealogy of palliative care in medicine. We try to understand how physicians take over social stakes, to promote new standards and new practices. With palliative care emerging we witness a transformation problem - setting around the end of life, establishing a particular approach to death in the medical world. Thus we intend to study the development of a specific doctrine about the end of life and the way in which this discipline has gradually forced itself a position in medicine. 
